\title{
Correction to: An improved correlation filter tracking method with occlusion and drift handling
}

\author{
Jun Liu ${ }^{1,2} \cdot$ Zhongqiang Luo $^{1,2,3} \cdot$ Xingzhong Xiong $^{1,2}$
}

Published online: 28 January 2020

c) Springer-Verlag GmbH Germany, part of Springer Nature 2020

Correction to: The Visual Computer

https://doi.org/10.1007/s00371-019-01776-6

Figures 1, 3 and Table 1 contain errors. The correct versions are given below.

The original article can be found online at https://doi.org/10.1007/ s00371-019-01776-6.

Zhongqiang Luo

zhongqiangluo@gmail.com

Jun Liu

liujun100911@163.com

Xingzhong Xiong

xzxiong@suse.edu.cn

1 Artificial Intelligence Key Laboratory of Sichuan

Province, Sichuan University of Science and Engineering,

Yibin 644000, China

2 School of Automation and Information Engineering, Sichuan University of Science and Engineering, Yibin 644000, China

3 Key Laboratory of Higher Education of Sichuan Province for Enterprise Informationalization and Internet of Things, Sichuan University of Science and Engineering, Yibin 644000, China 


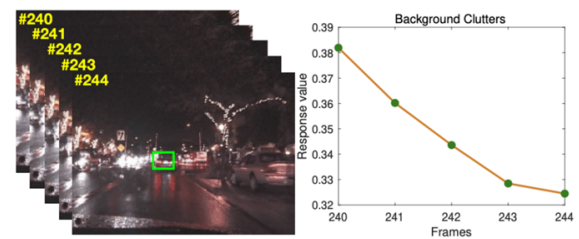

(a) CarDark

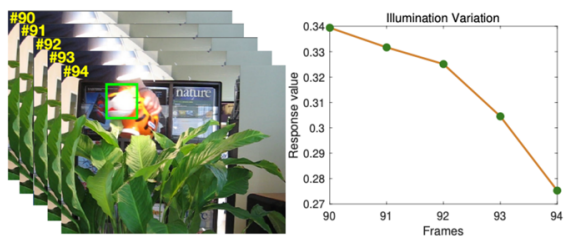

(b) Tiger 1

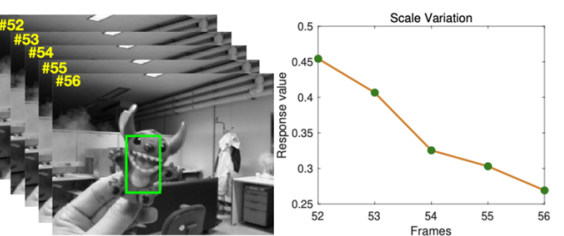

(c) Toy

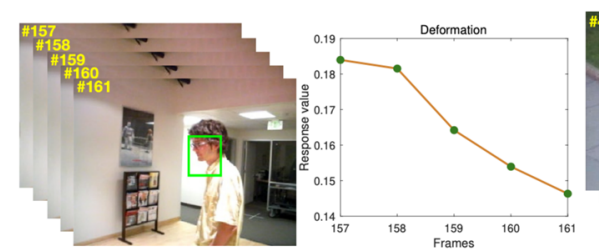

(d) David

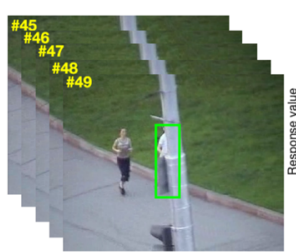

(e) Jogging 1

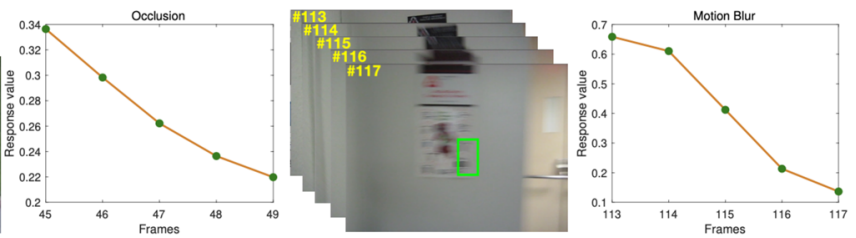

(f) BlurOwl

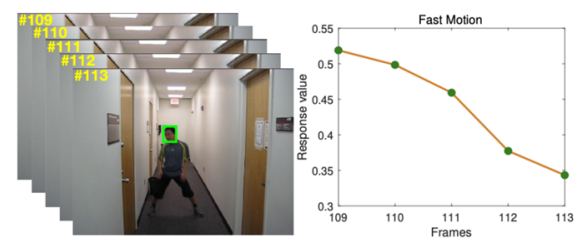

(g) Boy

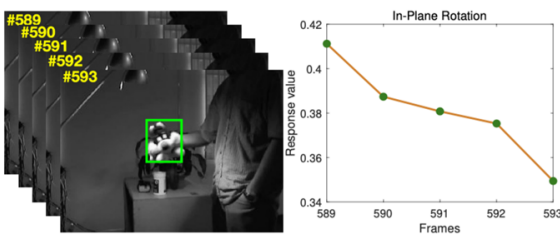

(h) Sylvester

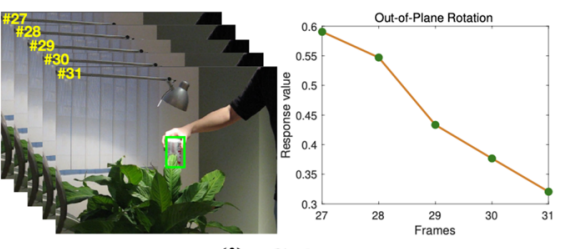

(i) Coke

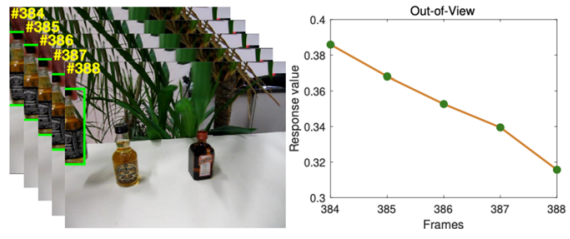

(j) Liquor

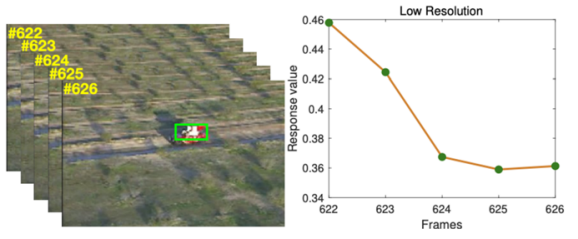

(k) RedTeam

Fig. 1 The trend of response curve in 11 challenging scenarios 


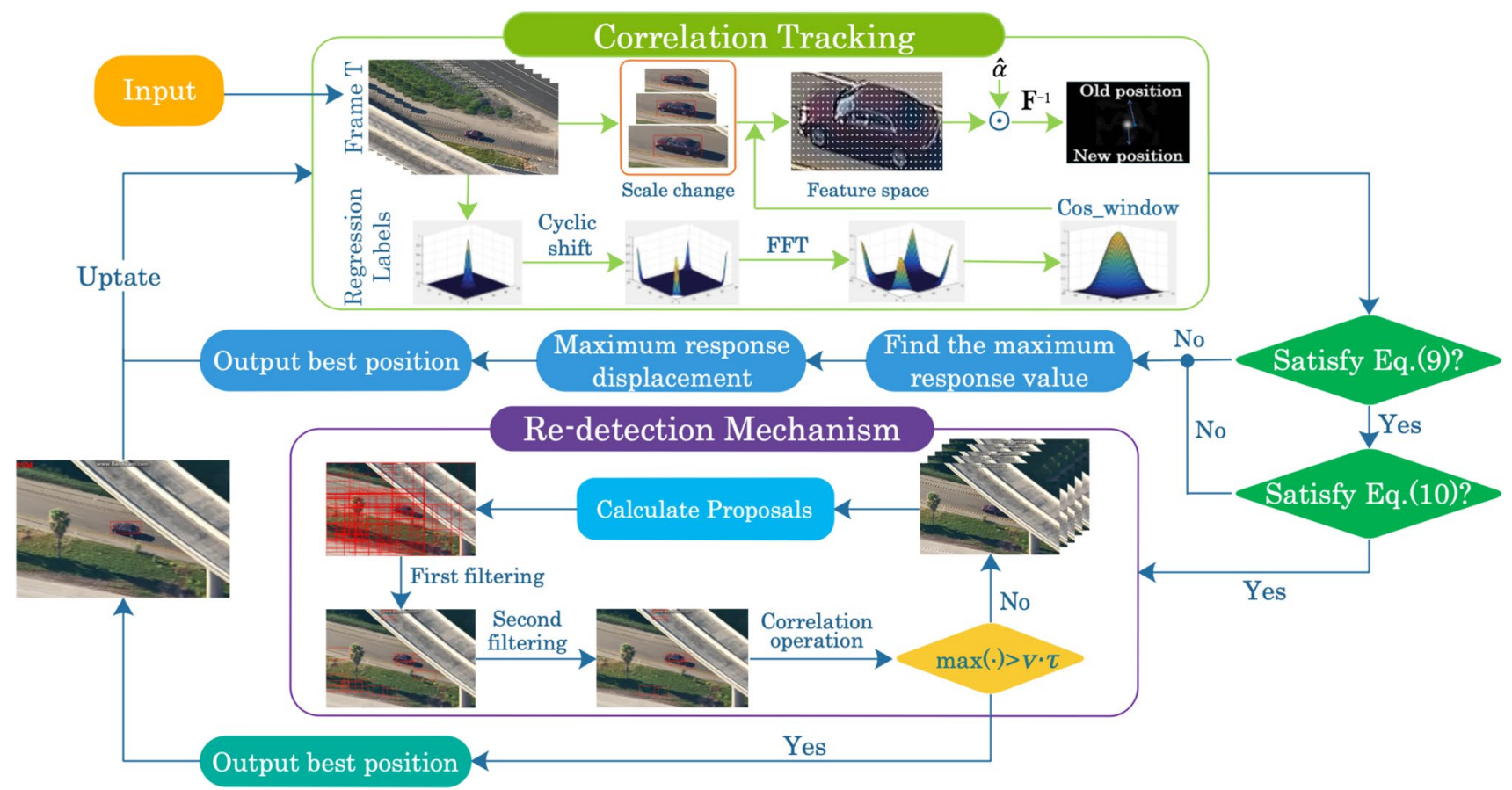

Fig. 3 The flowchart of proposed tracker. As shown in the figure, after the video sequence is input to the correlation tracking component, the corresponding response value is obtained, and then the criterion component will decide whether to enable the re-detection

Table 1 The second frame response value corresponding to each of the 11 sequences

\begin{tabular}{|c|c|c|c|c|}
\hline Sequence & Cardark & Boy & David & Toy \\
\hline$\tau$ & 0.604 & 0.750 & 0.844 & 0.922 \\
\hline Sequence & BlurOwl & Sylvester & Coke & Liquor \\
\hline$\tau$ & 0.840 & 0.934 & 0.813 & 0.937 \\
\hline Sequence & Jogging1 & & & RedTeam \\
\hline$\tau$ & 0.796 & 0.7 & & 0.608 \\
\hline
\end{tabular}

mechanism. If the re-detection mechanism is activated, the most reliable result is taken to initialize the tracking component and update the template

Publisher's Note Springer Nature remains neutral with regard to jurisdictional claims in published maps and institutional affiliations. 